\title{
论 文
}

\section{脉冲电流对 Al-Mg 合金力学性能和断口的影响}

\author{
范蓉 ${ }^{(1)}$, 赵坤民 ${ }^{(12) *}$, 任大金 ${ }^{(1)}$, ZIKRY Mohammed A. ${ }^{\text {(3) }}$ \\ (1) 大连理工大学汽车工程学院, 工业装备结构分析国家重点实验室, 大连 116024; \\ (2) 合肥工业大学工业与装备技术研究院，合肥 230601; \\ (3) Department of Mechanical and Aerospace Engineering, North Carolina State University, Raleigh 27695, USA \\ *E-mail: kmzhao@dlut.edu.cn \\ 收稿日期: 2016-03-15; 接受日期: 2016-04-29; 网络出版日期: 2016-06-22 \\ 国家自然科学基金(批准号: 11472072)和大连理工大学基本科研业务费(编号: DUT15ZD103)资助项目
}

摘要研究了高能量密度 $0.150 \mathrm{~J} / \mathrm{mm}^{3}$ 和低能量密度 $0.105 \mathrm{~J} / \mathrm{mm}^{3}$ 下, 不同脉冲电流参数对铝合金流动应力和 伸长率的影响. 铝合金在相同的能量密度下产生的温度一致, 由此探讨了电辅助成形中的非焦耳热效应. 结果 表明: 脉冲电流的引入能够降低铝合金的最大应力值, 提高伸长率. 相同能量密度下, 随着脉冲电流密度的增 加, 最大应力值保持不变, 但是脉冲电流引起的瞬时应力下降值增加, 且伸长率显著增加. 通过应力回复模型, 准确预测出脉冲电流引起的应力下降值. 高能量密度下试样拉伸后的断口㓞窝数量变少, 并且随着脉冲电流密 度的增加, 断口韧窝数量减少直至消失, 断口伸长率的提高也正是脉冲电流抑制了空洞的形核和长大所致.

关键词铝合金, 脉冲电流, 流动应力, 断口形貌, 伸长率

\section{1 引言}

铝合金由于其具有比强度高、密度小、耐腐蚀等 优点, 被广泛地应用到了汽车和航空航天工业领域, 以达到降低能耗, 减少尾气排放的目的 ${ }^{[1]}$. 然而, 铝 合金在室温下的塑性差, 成形较困难, 其应用受到了 限制 ${ }^{[2,3]}$. 电辅助成形技术是在金属成形过程中通入 电流, 实现降低变形抗力, 提高伸长率的作用 ${ }^{[4]}$. 早 期针对该技术的研究主要集中在高电流密度 $(>100$ $\left.\mathrm{A} / \mathrm{mm}^{2}\right)$ 下 $^{[5]}$, 为便于现代工业生产应用, 学者开始针 对材料在低电流密度 $\left(<100 \mathrm{~A} / \mathrm{mm}^{2}\right)$ 下的力学行为展
开研究. 然而, 部分研究表明只有通电时产生的焦耳 热对流动应力产生影响 ${ }^{[6,7]}$ (焦耳热效应)，且材料的 伸长率由于温度梯度的影响而降低, 不利于成形 ${ }^{[8]}$. 另外有研究表明额外的应力减少出现在相同焦耳热 不同电流密度的情况下, 不能单独地用焦耳热效应 解释 ${ }^{[9]}$. 目前, 尚未有报道对比研究铝合金在相同焦 耳热效应下的电辅助成形力学性能. 由于相同的能 量密度产生焦耳热相同 ${ }^{[10]}$ ，根据此特性，本文以 5754 铝合金为研究对象, 对比分析在相同的能量密 度下, 不同的脉冲电流参数对其流动应力和伸长率 的影响.

引用格式: 范蓉, 赵坤民, 任大釒, 等. 脉冲电流对 Al-Mg 合金力学性能和断口的影响. 中国科学: 技术科学, 2016, 46: 717-721

Fan R, Zhao K M, Ren D X, et al. Effect of pulse current on the mechanical properties and fracture behaviors of Al-Mg alloys (in Chinese). Sci Sin Tech, 2016, 46: 717-721, doi: 10.1360/N092016-00098 


\section{2 实验材料及方法}

实验材料为厚度为 $1 \mathrm{~mm}$ 的 $\mathrm{O}$ 态 $\mathrm{Al}-\mathrm{Mg}$ 系铝合 金板材, 其主要成分(质量分数)为 $\mathrm{Mg} 2.60 \%$ 3.60\%, $\mathrm{Si} \leqslant 0.40 \%, \mathrm{Cu} \leqslant 0.10 \%, \mathrm{Zn} \leqslant 0.20 \%, \mathrm{Mn} \leqslant 0.50 \%, \mathrm{Ti} \leqslant$ $0.15 \%, \mathrm{Cr} \leqslant 0.30 \%, \mathrm{Fe} \leqslant 0.40 \%$, 余量为 $\mathrm{Al}$. 将铝合金沿 着轧制方向加工成拉伸试样. 试验在卧式拉伸机上 进行, 通过脉冲直流电源在拉伸过程中分别通入不 同大小和频率的电流, 每组实验重复 3 次. 试验过程 中试样的温度分布由 FLIR A 325 实时监控. 为安全起 见, 通电拉伸过程中试样的应变采用非接触式应变 测量技术获得, 采用高分辨率数字相机记录拉伸过 程中试样表面散斑位移, 经后处理得到试样的实时 应变. 对于给定的脉冲参数, 初始能量密度由以下函 数给出 ${ }^{[10]}$ :

$$
j_{0}=\frac{J_{0}}{V}=\left(\frac{I}{A_{0}}\right)^{2} \rho_{e} t_{d},
$$

式中, $A_{0}$ 为材料的初始横截面积, $\rho_{e}$ 为铝合金的电阻 系数 $\left(4.6 \times 10^{-5} \Omega \mathrm{mm}\right) ; I$ 和 $t_{d}$ 分别为通入的脉冲电流 强度和持续时间. 为达到相同的能量密度, 单位横截 面积上的脉冲电流强度越高, 脉冲持续时间越短, 本 次试验参数见表 1 , 脉冲间隔时间保持一致为 $20 \mathrm{~s}$. 拉伸完成后, 切取试样断口在 S-4800 型扫描电子显 微镜下观察断口形貌.

\section{3 分析与讨论}

\section{1 不同能量密度下的温度和应力应变曲线}

监测拉伸过程中试样表面的最高温度的变化曲线 (图 1), 为观察方便，除了脉冲持续时间为 $1 \mathrm{~s}$ 的情况 外, 其余仅显示温度峰值. 在相同的能量密度下, 脉 冲作用产生的温度峰值保持不变. 在脉冲电流的作用

\section{表 1 拉伸过程中的脉冲电流参数}

\begin{tabular}{ccccc}
\hline & $\begin{array}{c}\text { 电流密度 } \\
\left(\mathrm{A} / \mathrm{mm}^{2}\right)\end{array}$ & $\begin{array}{c}\text { 脉冲持续 } \\
\text { 时间 }(\mathrm{s})\end{array}$ & $\begin{array}{c}\text { 脉冲间隔 } \\
\text { 时间 }(\mathrm{s})\end{array}$ & $\begin{array}{c}\text { 能量密度 } \\
\left(\mathrm{J} / \mathrm{mm}^{3}\right)\end{array}$ \\
\hline 1 组 & 68.0 & 0.5 & 20 & 0.105 \\
& 47.8 & 1.0 & 20 & \\
\multirow{4}{*}{ 组 } & 33.7 & 2.0 & 20 & \\
& 80.7 & 0.5 & 20 & 0.150 \\
& 57.0 & 1.0 & 20 & \\
& 40.4 & 2.0 & 20 & \\
\hline
\end{tabular}

下, 试样表面温度出现瞬间升高, 脉冲停止时迅速下 降. 拉伸初期, 试样变形引起材料温度略微升高; 随 着变形的增加, 每次脉冲引起的温度峰值不断升高, 这是由横截面积变小电流密度升高所致. 在相同的 能量密度下, 脉冲产生的温度峰值保持一致, 两种能 量密度下产生的初始温度峰值分别为 $95^{\circ} \mathrm{C}$ 和 $130^{\circ} \mathrm{C}$.

图 2 为能量密度为 0.105 和 $0.150 \mathrm{~J} / \mathrm{mm}^{3}$ 下的真 实应力应变曲线, 脉冲持续时间均为 $0.5 \mathrm{~s}$. 脉冲电流 作用的瞬间, 试样应力下降明显, 且能量密度值越高, 应力下降值越大. 一旦脉冲电流停止, 应力迅速回升 至屈服硬化直至下次脉冲到来. 和未施加电流下的 拉伸结果相比, 单次脉冲的应力回复值随着能量密度 值的增加而下降. 两种能量密度下，铝合金的最大应 力值由 $273.81 \mathrm{MPa}$ 分别减少至 258.35 和 $235.84 \mathrm{MPa}$, 伸长率由 $19.65 \%$ 分别增加至 $21.71 \%$ 和 $30.69 \%$.

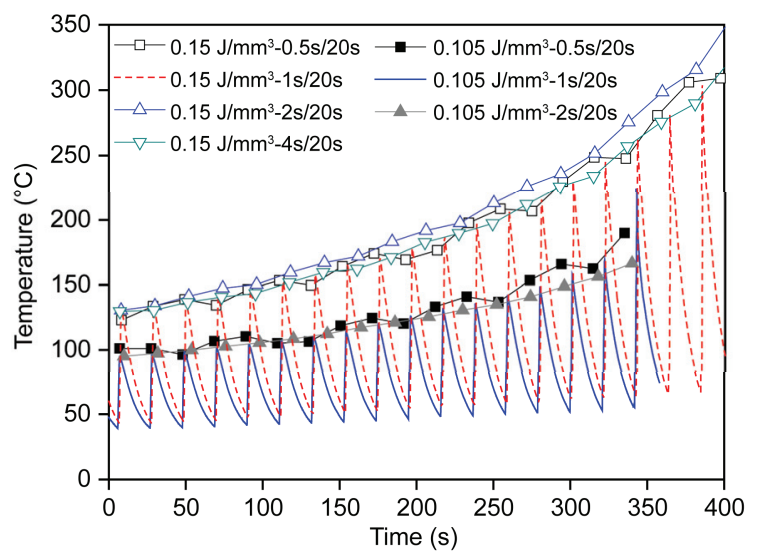

图 1 (网络版彩图)拉伸过程中不同脉冲参数下的温度随时 间变化曲线

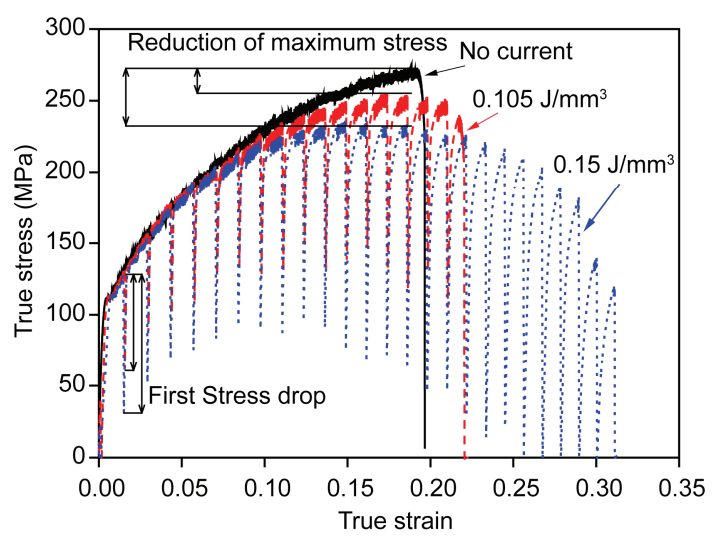

图 2 (网络版彩图)铝合金板材真实应力应变曲线(脉冲持 续时间为 $0.5 \mathrm{~s}$ ) 


\section{2 相同能量密度下的应力下降值}

试样在不同脉冲电流参数下引起的瞬时应力下 降值如图 3 所示. 在相同的脉冲电流密度下, 高能量 密度引起更加显著的应力下降. 在 $0.150 \mathrm{~J} / \mathrm{mm}^{3}$ 下, 脉冲电流密度从 28.6 上升到 $80.7 \mathrm{~A} / \mathrm{mm}^{2}, 5754$ 铝合 金的应力下降值由 7.44 增加到 $96.10 \mathrm{MPa}$. 可以看出, 相同能量密度下, 脉冲电流密度值越高引起的应力 下降值越大.

5754 铝合金的瞬时应力变化主要由两部分构成: 一方面, 电流产生焦耳热引起试样温度升高, 焦耳热 引起试样产生热膨胀, 加载状态下的试样出现应力 松弛, 应力松弛值 $\sigma_{\mathrm{T}}$ 与弹性模量 $E$ 成正比; 另一方面, 拉伸的持续进行引起试样变形增加, 进而导致拉伸 应力值 $\sigma_{\mathrm{M}}$ 升高, 当脉冲持续时间较长时, 应变增加 量接近甚至超过了热膨胀引起的应变值, 因此, 应力 升高值采用塑性加载计算得到, 新增应变可以通过 应变率和电流持续时间计算得出. 脉冲电流持续时 间内, 焦耳热引起的应力下降值大于相同时间内拉 伸产生的应力升高值. 综合这两方面因素, 脉冲电流 引起的应力下降值的计算公式可以表达为

$$
\sigma_{\text {drop }}=\sigma_{\mathrm{T}}+\sigma_{\mathrm{M}}=E \frac{j_{0}}{c \rho} \mu \alpha-K\left(\dot{\varepsilon} t_{d}\right)^{n},
$$

式中, $j_{0}$ 为脉冲电流能量密度, $c$ 为材料比热容, $E$ 为弹 性模量, $\dot{\varepsilon}$ 为应变率, $\mu, n, K$ 为经验系数(通过数据拟 合获得). 在相同的能量密度下, 脉冲电流密度越大, 应力下降值越高. 图 3 显示了不同脉冲参数下的初始 应力下降值的理论结果和试验结果, 吻合较好.

\section{3 相同能量密度下的最大应力值和伸长率}

图 4 为 5754 铝合金最大应力和伸长率随脉冲电 流密度的变化趋势. 和未通电下的拉伸结果相比, 能 量密度越高, 材料的最大应力值越低. 但是, 相同能 量密度下, 最大应力值随着电流密度的变化不明显, 即最大应力值不因电流密度的变化而变化.

低能量密度 $\left(0.105 \mathrm{~J} / \mathrm{mm}^{3}\right)$ 下, 5754 铝合金的伸长 率由未通电下的 $19.7 \%$ 提高到 $21.5 \%$, 随着脉冲电流 密度的增加, 伸长率保持一致不发生明显变化; 高能 量密度 $\left(0.150 \mathrm{~J} / \mathrm{mm}^{3}\right)$ 下, 5754 铝合金的伸长率由 $19.7 \%$ 显著提高到 $31.4 \%$ ，提高了 $40 \%$; 且在该能量 密度下, 脉冲电流密度值越大, 试样的伸长率越高. 一般认为, 伸长率的升高受温度影响. 图 5 为 5754 铝合金在不同温度下的伸长率, 温度通过连续电流

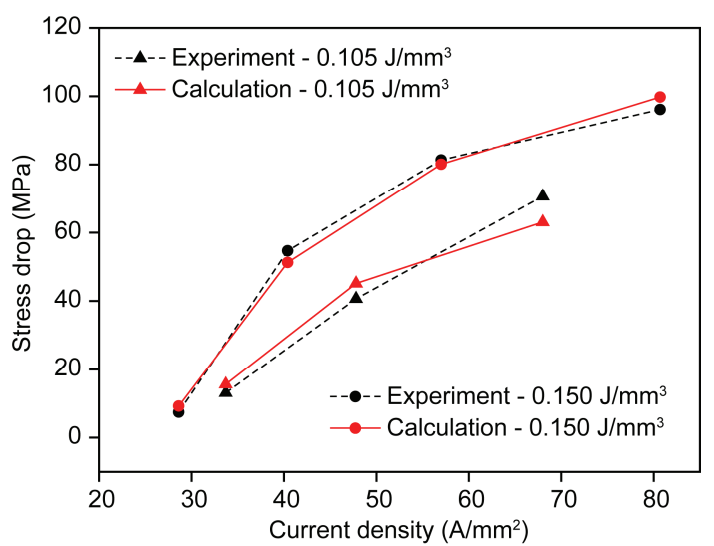

图 3 (网络版彩图)不同脉冲电流引起的瞬时应力下降值

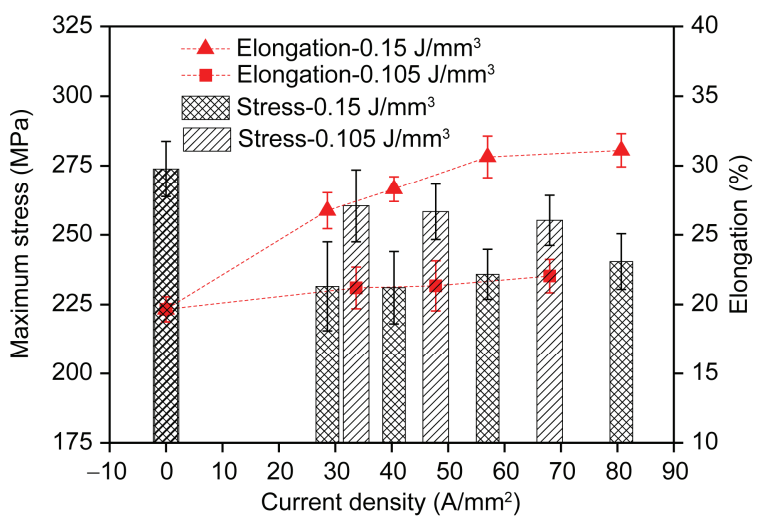

图 4 (网络版彩图)脉冲电流密度对铝合金最大应力和伸长 率的影响

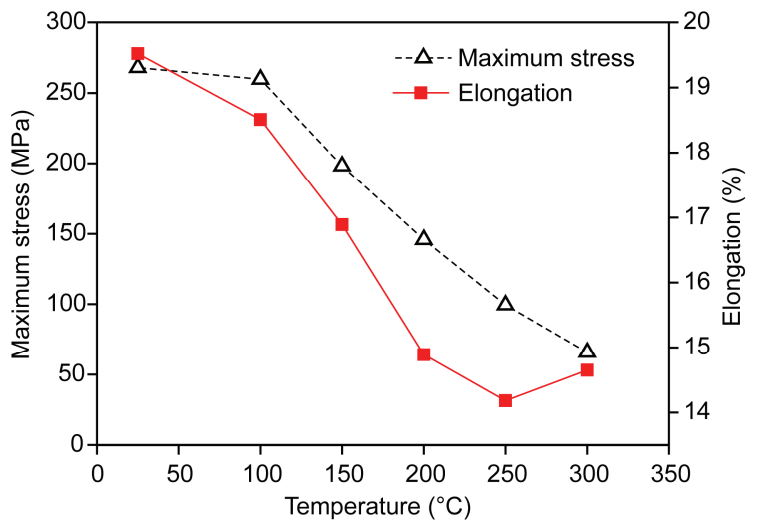

图 5 (网络版彩图)焦耳热对铝合金最大应力和伸长率的 影响

产生, 通过控制电流强度, 保证在拉伸过程中, 试样 表面的温度最高值保持不变. 可以看出, 试样的伸长 率反而随着目标温度的上升而下降，相同的现象出 
现在 $\mathrm{Mg}$ 合金等拉伸试验中 ${ }^{[8]}$. 这是由于拉伸时, 试 样中部的能量密度高, 产生的热量多, 因此, 局部温 度高于标距内其他位置的温度, 试样表面的温度梯 度使得该区域更易发生局部颈缩, 降低材料的伸长 率. 因此, 焦耳热并不是 5754 铝合金伸长率增加的 原因.

当电流通过金属导体时, 金属中的自由电子在 电场的作用下发生定向运动, 运动电子流与位错产 生交互作用, 促进位错运动, 加速位错的滑移和攀移, 减小位错缠结, 提高位错的可动性 ${ }^{[11,12]}$. 高的电流密 度, 加快电子位错的运动速度, 并且随着电流密度的 增加, 运动电子流速度越快, 因此, 促使位错穿越障 碍的能力越高. 高能量密度 $\left(0.150 \mathrm{~J} / \mathrm{mm}^{3}\right)$ 下, 高的脉 冲电流密度能够引起材料塑性变形的明显增加, 这 种现象在低能量密度 $\left(0.105 \mathrm{~J} / \mathrm{mm}^{3}\right)$ 下并不明显. 可以 认为, 当能量密度未达到一定值时, 即使电流密度很 高, 材料的塑性增加也不明显. 因此, 为了提高 5754 铝合金的塑性变形能力, 能量密度和脉冲电流密度 二者缺一不可.

\section{4 断口微观结构}

图 6 所示为不同脉冲电流参数下的试样断口形 貌. 可以看出, 没有施加电场的拉伸断口呈现明显的 㓞窝, 且韧窝大而深, 为典型的韧性断裂. 施加脉冲 电流的试样断口韧窝明显减少, 且脉冲电流参数不 同, 断口形貌不同. 低电流密度 $28.6 \mathrm{~A} / \mathrm{mm}^{2}$ 下的断口 出现大量的沿晶断裂, 且韧窝明显减少, 这与高温下
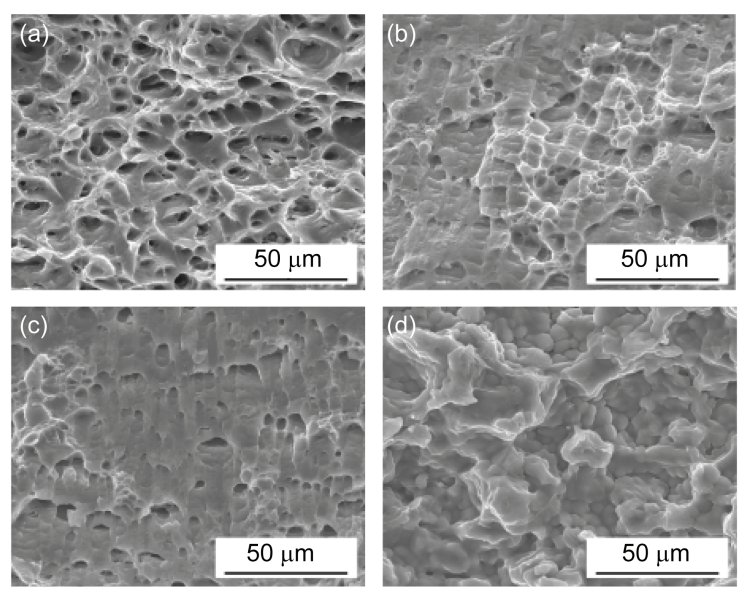

图 6 高能量密度下不同脉冲电流参数下的断口形貌

(a) 无; (b) $28.6 \mathrm{~A} / \mathrm{mm}^{2}$; (c) $40.4 \mathrm{~A} / \mathrm{mm}^{2}$; (d) $80.7 \mathrm{~A} / \mathrm{mm}^{2}$
的断裂形式类似, 当电流密度上升到 $40.4 \mathrm{~A} / \mathrm{mm}^{2}$ 时, 断口主要为小而浅的韧窝, 随着电流密度的进一步 升高, 试样断口出现片状层, 并有不均匀杂质析出.

多晶体金属在断裂过程中, 主要经历空洞的形 核、长大、相互聚合和链接, 进而导致断裂. 材料内 部形成空洞, 且空洞一般位于三叉晶界或者第二相 粒子中, 在这些地方, 晶界滑移引起局部应力集中, 原子扩散或者位错运动来调节和消除这种应力集中 现象. 如果晶界滑移速度超过了调节速度, 就会引起 空洞形核, 断口上的空洞通过塑性变形中的空洞聚 集连接形成. 在无脉冲电流的情况下, 塑性变形产生 的空洞多, 韧窝密集. 脉冲电流可以降低再结晶激活 能, 提高晶界处的晶核形核率, 细化再结晶晶粒, 并 抑制和推迟空洞的形成 ${ }^{[13]}$, 使形成韧窝并导致断裂 的所需塑性变形有所增加, 从而提高了材料的塑性. 同时, 当高密度的电流在极短时间内被传输到材料 内部时, 原子的随机热运动在脉冲电流瞬时冲击力 作用下获得足够的动能离开平衡位置, 显著提高内 部原子扩散能力, 帮助打开金属的位错缠结, 克服位 错滑移面上的阻碍, 提高材料塑性变形的能力 ${ }^{[14]}$. 即 使在相同的焦耳热下, 随着单次脉冲电流密度峰值 的增加, 韧窝的形成也随之退后, 数量也随之减少. 脉冲电流密度的增加促进了位错的运动和原子扩 散 ${ }^{[15]}$, 更有效缓解了晶界滑移产生的局部集中应力, 抑制了空洞的形核和长大, 从而使试样的伸长率进 一步得到提高.

\section{4 结论}

脉冲电流产生的能量密度越高, 5754 铝合金的 流动应力越低, 伸长率越高; 在相同的能量密度下, 脉冲电流密度越高, 脉冲引起的瞬时应力下降值越 大. 在低能量密度 $\left(0.105 \mathrm{~J} / \mathrm{mm}^{3}\right)$ 下, 5754 铝合金的最 大应力和伸长率不随脉冲电流密度的变化而明显变 化; 在高能量密度 $\left(0.150 \mathrm{~J} / \mathrm{mm}^{3}\right)$ 下, 5754 铝合金的最 大应力值不变, 但伸长率随着脉冲电流密度的增加 而明显增加. 5754 铝合金伸长率的提高由脉冲能量密 度和脉冲电流密度两者共同决定. 在低能量密度下, 伸长率变化很小, 只有当能量密度达到一定值时, 脉 冲电流密度越高, 伸长率越大. 通过观察 5754 铝合 金的拉伸断口形貌，发现脉冲电流的引入抑制了空 洞的形核和长大, 使得成形性能得到显著提高. 
1 Wang H, Luo Y, Peter F, et al. Warm forming behavior of high stregnth aluminum alloy AA7075. T Nonferr Metal Soc, 2012, 22: 1-7

2 Ozturk F, Toros S, Pekel H. Evaluation of tensile behaviour of 5754 aluminium-magnesium alloy at cold and warm temperatures. Mater Sci Tech-Lond, 2009, 25: 919-924

3 曹零勇, 郭明星, 崔华, 等. 汽车用 6111 铝合金板材力学性能和织构研究. 材料热处理学报, 2013,34: 118-123

4 Song H, Wang Z J, He X D. Improving in plasticity of orthorhombic $\mathrm{Ti}_{2} \mathrm{AlNb}$-based alloys sheet by high density electropulsing. Trans Nonferrous Met Soc China, 2013, 23: 32-37

5 郑明新, 朱永华, 唐国翌, 等. 关于电塑性拔丝和其结构演变的讨论. 清华大学学报(自然科学版), 1998, 38: 28-32

6 Kinsey B, Cullen G, Jordan A, et al. Investigation of electroplastic effect at high deformation rates for 304SS and Ti-6Al-4V. CIRP Ann-Manuf Techn, 2013, 62: 279-282

7 Magargee J M F, Cao J. Characterization of flow stress for commercially pure titanium subjected to electrically-assisted deformation. J Eng Mater Technol, 2013, 135: 041003-1-10

8 䨠振球, 问否, 董湘怀. AZ31 镁合金与 DP980 高强钢的纯电塑性效应实验研究. 热加工工艺, 2015, 44: 31-36

9 Mai J, Peng L, Lin Z, et al. Experimental study of electrical resistivity and flow stress of stainless steel 316L in electroplastic deformation. Mat Sci Eng A-Struct, 2011, 528: 3539-3544

10 Roh J H, Seo J J, Hong S T, et al. The mechanical behavior of 5052-H32 aluminum alloys under a pulsed electric current. Int J Plasticity, 2014, 58: 84-99

11 Yao K F, Wang J, Zheng M, et al. A research on electroplastic effects in wire-drawing process of an austenitic stainless steel. Scripta Mater, 2001, 45: 533-539

12 姚可夫, 王沛玉. 脉冲电流对金属材料塑性变形和组织结构与性能的影响. 机械强度, 2003, 25: 340-342

13 樊慧君, 陈国清, 周文龙, 等. 直流匀强电场作用下镍钛合金的高温变形行为. 中国有色金属学报, 2006, 16: 1012-1018

14 Qiu S B, Yao K F. Crystallization behavior of $\mathrm{Zr}_{41} \mathrm{Ti}_{14} \mathrm{Cu}_{12.5} \mathrm{Ni}_{10} \mathrm{Be}_{22.5}$ bulk metallic glass under the action of high-density pulsing current. J Non-Cryst Sol, 2008, 354: 3520-3524

15 刘志义, 刘冰, 邓小铁, 等. 脉冲电流对 2091Al-Li 合金超塑变形机理的影响. 金属学报, 2000, 36: 944-954

\title{
Effect of pulse current on the mechanical properties and fracture behaviors of Al-Mg alloys
}

\author{
FAN Rong ${ }^{1}$, ZHAO KunMin ${ }^{1,2}$, REN DaXin $^{1} \&$ ZIKRY Mohammed A. ${ }^{3}$ \\ ${ }^{1}$ School of Automotive Engineering, State Key Lab of Structural Analysis for Industrial Equipment, Dalian University of Technology, Dalian \\ 116024, China; \\ ${ }^{2}$ Industrial and Equipment Technology Institute, Hefei University of Technology, Hefei 230601, China; \\ ${ }^{3}$ Department of Mechanical and Aerospace Engineering, North Carolina State University, Raleigh 27695, USA
}

The influence of pulse electric current to aluminum's flow stress and elongation under high electric energy density $\left(0.150 \mathrm{~J} / \mathrm{mm}^{3}\right)$ and low energy density $\left(0.105 \mathrm{~J} / \mathrm{mm}^{3}\right)$ are investigated. Since the temperatures generated by electric current are identical under same energy density with different electric conditions, non-Joule heating effect under electrically assisted forming can be proven. The results show that pulse current help to reduce aluminum's flow stress and increase its elongation. Under the same energy density, as the density of pulse current increased, although the maximum flow stress remains unchanged, the instant stress drop due to pulse current increases as well as its elongation. Stress recovery model is proposed to estimate the stress drop under tension. The number of fracture dimple continues to decrease until completely disappearance as the density of pulse current increases; the increase of elongation results from the suppression of the nucleation and growth of voids by pulse current.

aluminum alloy, pulse current, flow stress, fracture morphology, elongation

doi: $10.1360 / \mathrm{N} 092016-00098$ 